\title{
SINGLE PHOTON IONISATION MASS SPECTROMETRY USING LASER-GENERATED VACUUM ULTRAVIOLET PHOTONS
}

\author{
NICHOLAS P. LOCKYER and JOHN C. VICKERMAN \\ Department of Chemistry, UMIST, P. O. Box 88, \\ Manchester M60 1QD, U.K.
}

(Received 24 March 1997)

This paper provides an overview of the method of single photon ionisation mass spectrometry. A review of the theory of frequency upconversion using third-order 4wave sum mixing in isotropic media and experimental results of third harmonic generation (THG) using a frequency tripled $355 \mathrm{~nm}$ Nd:YAG pump source are presented. Vacuum Ultra-violet (VUV) photons of wavelength $118 \mathrm{~nm}$ are detected in an acetone ionisation chamber. The emphasis of this paper is on the practical aspects of generating and detecting the VUV photons and using them for single photon ionisation (SPI) in the ion source of a mass spectrometer. Optimum gas pressures for THG in Xe and $\mathrm{Xe} / \mathrm{Ar}$ mixtures are established. For a pump beam of well defined mode structure the optimum gas pressures are in excellent agreement with theory. The major loss mechanism is attributed to re-absorption of VUV by the tripling gas. SPI mass spectra of hexane and the biomolecule valyl-valine are presented illustrating the power of the technique.

Keywords: Vacuum ultra-violet; third harmonic generation; 4-wave sum mixing; phase matching; single photon ionisation

\section{INTRODUCTION}

Single photon ionisation mass spectrometry is a technique which promises to provide a highly sensitive, non-selective analytical method for a wide range of molecular systems. There are an increasing number of reports of the application of single photon (VUV) ionisation mass spectrometry, which indicate that the technique provides a greater 
molecular ion intensity and less fragmentation than both electron impact and many multiphoton ionisation schemes [1, 2, 3]. The generation of coherent VUV light is described in a variety of accounts of frequency up-conversion techniques scattered throughout the physics literature. Reviews on this subject have been given by Delone $e t$ al. [4] and by L'Huillier et al. [5]. This paper seeks to bring together the relevant theory and a systematic account of experiments to generate a practical source of VUV for integration into a Time-of-Flight Mass Spectrometer for ionisation of sputtered organic species.

\section{Theory}

Single photon ionisation (SPI) removes the nonlinearity present in multiphoton ionisation (MPI) schemes. The exploitation of nonlinear optics however remains the most convenient method for conversion of existing primary laser lines to wavelengths in the VUV, which are suitable for SPI of a wide range of molecular species.

Nonlinear optical effects are so called because the response of the media giving rise to them depends on the second- and higher-order powers of the optical fields acting on them. They occur due to energy transfer arising from wave interactions between propagating waves via coupling coefficients known as optical susceptibilities. An incident electric field $E_{i}$ induces a polarisation $(P)$ in a medium such that

$$
P=\chi^{(1)} E_{i}+\chi^{(2)} E_{i}^{2}+\chi^{(3)} E_{i}^{3} \ldots
$$

where $\chi^{(n)}$ is the $n^{\text {th }}$-order susceptibility of the medium. If the magnitude of the incident field is sufficiently large the induced polarisations will generate observable optical fields at harmonic frequencies of the incident field. Harmonic generation whereby $N_{\gamma}$ photons of frequency $(\omega)$ are converted to $\gamma^{\prime}$ photons of frequency $(N \omega)$ by a nonlinear medium $(X)$ is described by

$$
N_{\gamma}(\omega)+X=\gamma^{\prime}(N \omega)+X
$$

In centrosymmetric media the lowest order term of eq. (1) contributing to frequency mixing is $\chi^{(3)}$ and the dominant harmonic generation 
process is normally third-order in the number of incident photons. The power $I(3 \omega)$ generated at the third harmonic frequency is given by [6]

$$
I(3 \omega) \propto N^{2}\left|\chi_{a}^{(3)}(\omega)\right|^{2} I(\omega)^{3} F(L, b, \Delta k)
$$

$F$ is a geometrical factor which reflects the net phase relationship between microscopic individual third-harmonic wavelets generated in different regions of the nonlinear medium of length $L$ and number density $N$.

\section{Phase Matching}

If harmonic generation is to be efficient $F$ must be controlled such that complete destructive interference does not occur over the wave interaction length. In the case of incident plane-waves of wave vector $k(\omega) \mathbf{e}_{1}$ the fundamental and third harmonic wavelets are collinear and their phase velocities are matched. In this case phase matching is achieved when the wave vector mismatch $\Delta k=k(3 \omega)-3 k(\omega)$ is zero as shown in Figure 1(a), where the magnitude of the wave vector $k(\omega)$ is $2 \pi n(\omega) / \lambda_{0}$, where $n(\omega)$ is the refractive index of the tripling medium at frequency $\omega$ and $\lambda_{0}$ is the vacuum wavelength of the pump beam. This condition is met by ensuring that the refractive indicies of the tripling medium at frequencies $\omega$ and $3 \omega$ are equal.

To obtain sufficient photon densities it is often necessary to focus the pump beam. The degree of focusing is reflected in the confocal parameter $b=k w_{0}$, where $w_{0}$ is the minimum beam waist at the focus.

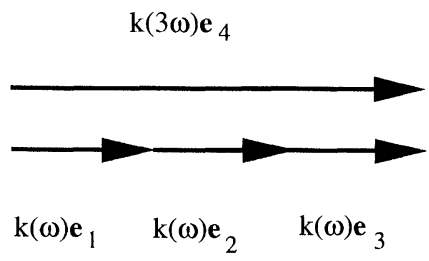

(a)

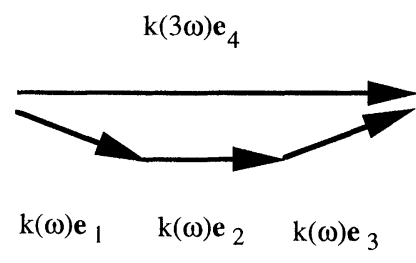

(b)

FIGURE 1 Wave vector diagrams for THG showing conservation of momentum under (a) plane-wave and (b) focused geometries [7]. 
If the fundamental beam is focused, then a phase-slip of $\pi$ will occur between the pump beam and the third harmonic beam at the focus location. In addition, the three unit vectors $\mathbf{e}_{i}$ will have slightly different direction (Fig. 1(b)). Conservation of wave vector momentum requires that the condition $k(3 \omega) \mathbf{e}_{4}=k(\omega) \mathbf{e}_{1}+k(\omega) \mathbf{e}_{2}+k(\omega) \mathbf{e}_{3}$ is met and conservation of energy requires that $\omega_{3}=3 \omega_{0}$. As a consequence, $k(3 \omega)<3 k(\omega)$ and phase-matching is achieved when $\Delta k$ is negative. To satisfy the condition $\Delta k<0$ and conserve energy requires that $n(3 \omega)<n(\omega)$, which is termed negative or anomalous dispersion. The requirement of negative dispersion limits the choice of media in which a given frequency may be tripled. In general, localised regions of negative dispersion are to be found on the high frequency side of a real onephoton absorption.

In a single component gaseous medium the phase mismatch is proportional to the number density of the nonlinear medium and is optimised by varying the pressure of the gas. In this case the phase matching factor $F$ is dependent on $N$. From eq. (3) it can be seen that the maximum third harmonic power is obtained by maximising $N^{2} F$. For simplicity a second power coefficient $G$ is defined as $G=(b \Delta k)^{2} F$. In a single component third harmonic power is maximised when $G$ is maximised.

If the number density of the negatively disperse component is kept constant, phase matching can be optimised by adding a second, positively disperse medium or by tuning through the dispersion curve. In a medium consisting of more than one component, only one of which necessarily contributes to the nonlinearity, each will contribute to $b \Delta k$, which is independent of the total number density but dependent on the ratio of components. The number density of the nonlinear medium $N$ and the phase matching factor for the mixture $F$ are independent in this case and the conversion efficiency maximises where $F$ maximises.

\section{Effects of Spatial Modes}

A fundamental laser mode $\mathrm{TEM}_{p 1}$ will generate a third harmonic beam consisting of a superposition of spatial modes with angular mode 31 and radial mode number in the range $0-3 p$ (except $3 p-1$, which is zero for all $b \Delta k$ ) [8]. The phase matching coefficient $F$ 
represents an integration of the amplitudes $F(\rho)$ of the different radial modes in the third harmonic beam (where $\rho=0$ to $p_{1}+p_{2}+p_{3}$ ).

$$
F=\sum_{\rho=0}^{p_{1}+p_{2}+p_{3}}|F(\rho)|^{2}
$$

A TEM $_{00}$ fundamental $\left(p_{1}+p_{2}+p_{3}=0,1=0\right)$ gives a phase matching coefficient $F(0)=(-b \Delta k) \exp (0.5 b \Delta k)$. Applying eq. (4) gives the full expression for $F$ in a nonlinear medium of length $L$ in the limit of tight focusing $(b \ll L)$,

$$
\begin{array}{cc}
F(L, b, \Delta k)=(\pi b \Delta k)^{2} \exp (b \Delta k) & \Delta k<0 \\
0 & \Delta k>0
\end{array}
$$

For a $\mathrm{TEM}_{00}$ pump beam third harmonic power displays a singlepeaked dependence on $b \Delta k$ (Fig. 2). In a single component medium the THG conversion efficiency is optimised (maximum $G$ ) at

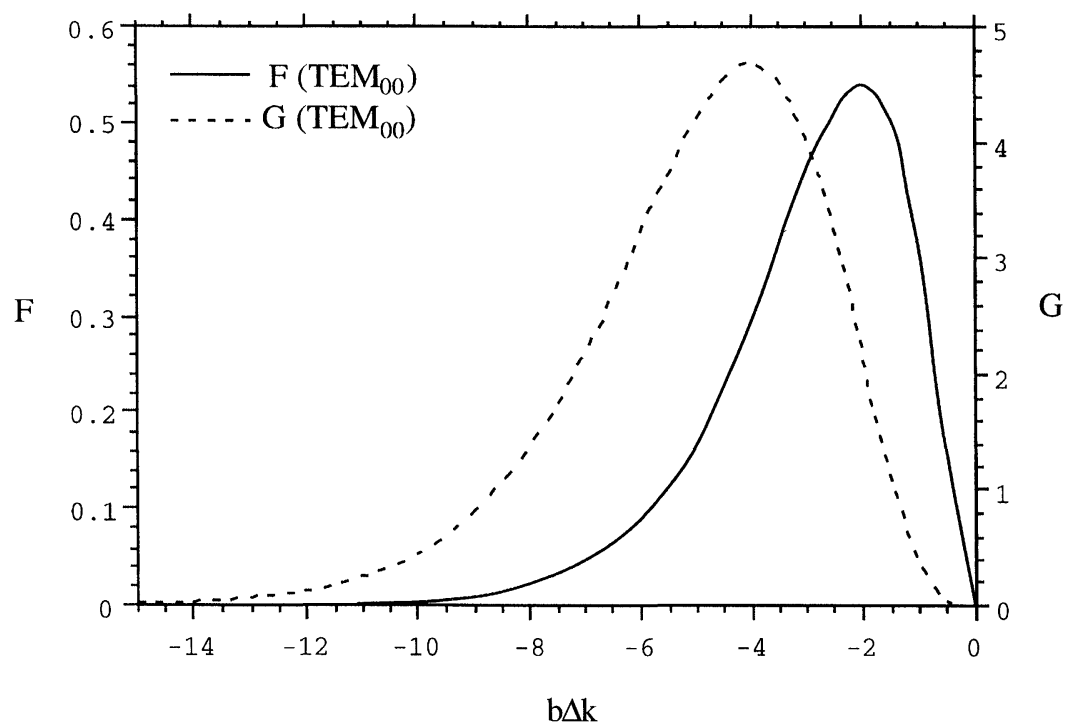

FIGURE 2 Power coefficients as a function of $b \Delta k$ for TEM $_{00}$ mode in a single component medium (broken line) and multi-component medium (solid line). 
$b \Delta k=-4$. In a multi-component medium the tripling efficiency is optimised (maximum $F$ ) at $b \Delta k=-2$.

Fundamental beams with non-zero radial modes $(p>0)$ generate harmonics with a mixture of radial modes, the power coefficients $F$ and $G$ having a multipeaked dependence on $b \Delta k$. The TEM ${ }_{10}$ mode generates harmonic fields displaying mode structures TEM $\mathrm{T}_{00}, \mathrm{TEM}_{10}$ and $\mathrm{TEM}_{30}$. The mode coefficients $F(\rho)$ are expressed below in terms of $|a|=0.5 b \Delta k$ [8]. The dependence of $F$ and $G$ on $b \Delta k$ is shown in Figure 3.

$$
\begin{aligned}
& F(0)=-\frac{2}{9}\left(4|a|-12|a|^{2}+|a|^{3}-\frac{4}{3}|a|^{4}\right) \exp (-|a|), \\
& F(1)=\frac{2}{9}\left(3|a|-6|a|^{2}+2|a|^{3}\right) \exp (-|a|), \\
& F(2)=0 \\
& F(3)=\frac{4}{9}|a| \exp (-|a|) .
\end{aligned}
$$

Compared to a TEM $_{00}$ pump field, the maximum value of $F$ is smaller and that of $G$ larger with a TEM 10 fundamental mode. Both maxima occur at higher values of $|b \Delta k|$, at 11.5 and 13.0 respectively.

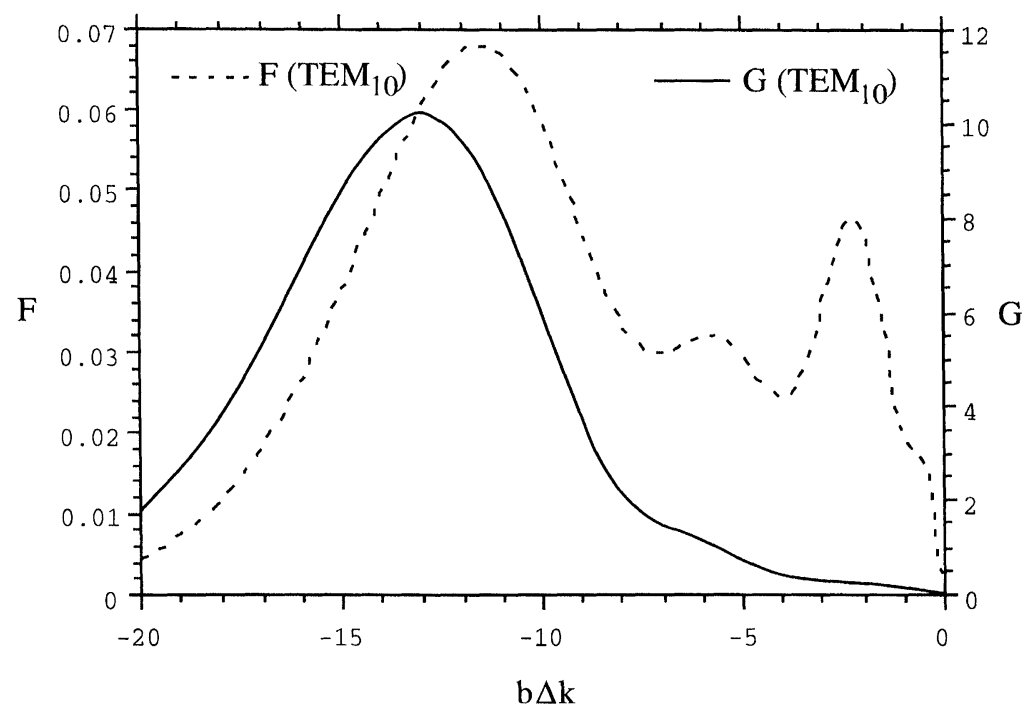

FIGURE 3 Power coefficients as a function of $b \Delta k$ for $\mathrm{TEM}_{10}$ mode in a single component medium (solid line) and multi-component medium (broken line). 


\section{GENERATION OF VUV PHOTONS}

\section{Experimental}

The primary laser source used in this work was a solid state Nd:YAG laser (Spectra-Physics DCR-11). Using an HG-2 harmonic generating unit the third harmonic of the Nd:YAG $(355 \mathrm{~nm})$ was obtained by generating second harmonics in a $\mathrm{KD}^{*} \mathrm{P}$ doubling crystal and mixing it with the residual fundamental in a second $\mathrm{KD}^{*} \mathrm{P}$ crystal. The third harmonic was then used as a pump beam to generate $118 \mathrm{~nm}$, the ninth harmonic of the Nd:YAG laser. The maximum pulse energy at $355 \mathrm{~nm}$ was $50 \mathrm{~mJ}$ at $10 \mathrm{~Hz}$ in a $5 \mathrm{~ns}$ pulse. Xenon gas, which is negatively disperse in the region $117.2-119.2 \mathrm{~nm}$ due to the transitions $(5 p-5 d)$ [9], was used as a tripling medium. Phase-matching was investigated with argon, which is positively disperse in this region.

Studying the VUV generation process by monitoring the SPI signal detected by the mass spectrometer introduces added complications. In order to obtain a reliable measure of the VUV flux, the ion extraction efficiency of the ToF must be optimised for each measurement. Due to the many parameters involved in determining the extraction efficiency, the amount of time required for the optimisation process makes this approach impracticable if the tripling process is to be studied in depth. For this reason it was felt necessary to design and construct an apparatus to generate and detect VUV photons on the "bench-top", without requiring the mass spectrometer. This apparatus is shown in Figure 4.

The tripling cell consists of a six-way stainless steel cross piece, 10 $\mathrm{cm}$ in length. Extension pieces allow the length of the cell to be increased to $70 \mathrm{~cm}$. The $355 \mathrm{~nm}$ laser light is focused in the centre of the cell through a fused silica window with a plano-convex fused silica lens. Xenon (99.996\%) and argon (99.999\%) are admitted to the cell through a fine leak valve. The pressure inside the cell is measured with a capacitance manometer of $1-1000$ torr range (MKS Baratron 221A) and a Penning gauge (Edwards CP25K).

The detection cell consists of a $13 \mathrm{~cm}$ long PVC tube containing two $10 \mathrm{~cm} \times 1.5 \mathrm{~cm}$ parallel-plate stainless steel electrodes $2 \mathrm{~cm}$ apart. One electrode is held at earth potential and the other connected to a digital picoammeter (Keithley model 485 ) biased at +30 VDC. The ionisation 


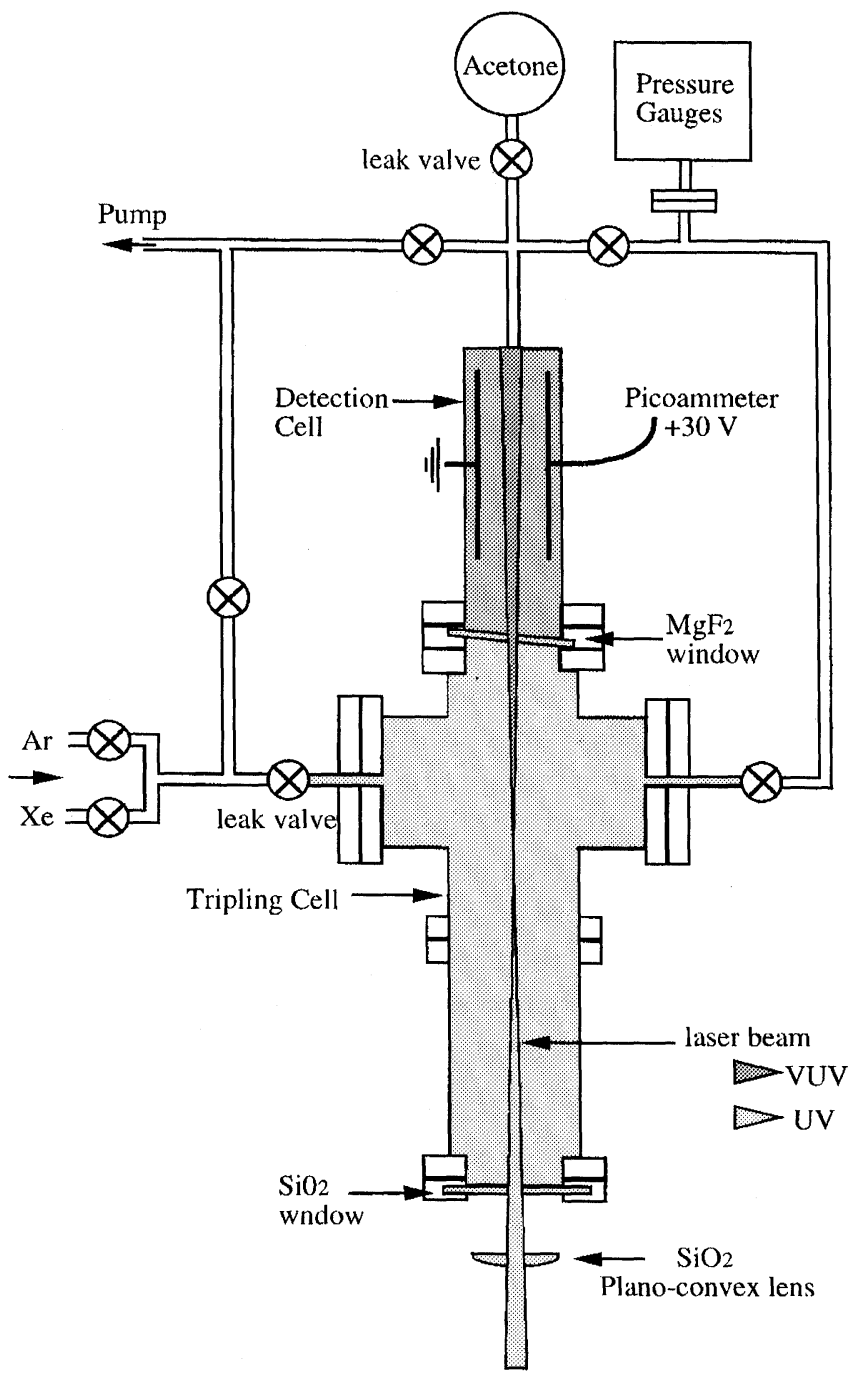

FIGURE 4 Schematic diagram of tripling cell for THG and acetone ionisation cell for VUV detection.

cell is operated with a 10 torr fill of acetone. The ionisation potential of acetone is $9.69 \mathrm{eV}(127.9 \mathrm{~nm})$ which is just below the energy of the VUV photons generated. Assuming that multiphoton ionisation of acetone in the diverging UV beam and photoelectron production at 
the chamber wall are negligible the current of photoelectrons attracted to the anode will give a direct measure of the VUV flux. These assumptions are supported by the observation that background photocurrents were $<1 \mathrm{pA}$ in the absence of a tripling gas. The two cells are separated by a $0.5 \mathrm{~cm} \times 2.5 \mathrm{~cm}$ diameter magnesium fluoride window and each can be independently pumped by a $170 \mathrm{~L} \mathrm{~s}^{-1}$ turbomolecular pump (Balzers TPU 170) backed with a rotary pump (Edwards M8).

VUV is readily absorbed by nitrogen so it is very important to minimise the residual air content of the tripling apparatus. The tripling cell was evacuated to a pressure $<10^{-5}$ torr prior to admitting the tripling gas. When using a two component mixture, a magnetic stirring bar can be used to aid mixing and to help minimise composition gradients within the cell.

\section{Results and Discussion}

A series of experiments were performed to optimise the sum-frequency process for generating $118 \mathrm{~nm}$ photons, some of these are described below.

\section{Effect of Confocal Parameter b on Conversion Efficiency}

In order to make most use of the available pump power it is necessary to establish the optimum focusing conditions. The effect on the conversion efficiency of changing the confocal parameter $b$ was investigated. This was done by changing the focal length $\left(f_{355}\right)$ of the lens which focuses the $355 \mathrm{~nm}$ beam into the tripling cell. An $f_{355}=$ $15 \mathrm{~cm}$ lens and an $f_{355}=50 \mathrm{~cm}$ lens were used for this experiment. The confocal parameter $(b)$ is given by

$$
b=\left(2 \pi w_{0}^{2} n\right) / \lambda_{1}
$$

where

$$
\begin{aligned}
n & =1(\text { refractive index for Xe at } 355 \mathrm{~nm}) \\
\lambda_{1} & =3.55 \times 10^{-4} \mathrm{~mm} \\
w_{0} & =\text { beam waist radius in } \mathrm{mm} \\
& =f \theta
\end{aligned}
$$


and [10],

$$
\theta=\frac{1}{2} K\left(n_{1}\right)(D / f)^{3}+1.22 \lambda_{1} / n D
$$

where

$$
\begin{aligned}
D & =6 \mathrm{~nm}(\text { diameter of } 335 \mathrm{~nm} \text { beam at lens }) \\
n_{1} & =1.461\left(\text { for } \mathrm{SiO}_{2} \text { at } 355 \mathrm{~nm}\right) \\
K\left(n_{1}\right) & =\frac{1}{32}\left(n_{1}-1\right)^{-2}\left(n_{1}^{2}-2 n_{1}+\frac{2}{n_{1}}\right)
\end{aligned}
$$

From eq. (6) the confocal parameters for the $f_{355}=15 \mathrm{~cm}$ and $f_{355}=50$ $\mathrm{cm}$ lenses were calculated as $0.2 \mathrm{~cm}$ and $2.3 \mathrm{~cm}$ respectively. The calculated minimum beam waist radii were $11 \mu \mathrm{m}$ and $36 \mu \mathrm{m}$ respectively.

The phase matching curves for THG in pure Xe using $f_{355}=15 \mathrm{~cm}$ and $f_{355}=50 \mathrm{~cm}$ lenses are shown in Figures $5(\mathrm{a})$ and (b) respectively. The use of the longer focal length lens improves the maximum conversion efficiency by a factor of two for equal UV pulse energies, even though the pump power density at the focus is an order of magnitude less compared to the shorter focal length lens.

Using a longer focal length lens for THG in gas cells means that i) the focal volume, where nonlinear interactions occur with the highest intensity, expands in size and ii) the beam divergence is reduced. This means that the number of scattering centres that can contribute to THG increases and higher pulse energies can be used without inducing breakdown in the tripling medium. However, the distance between the focus and the end window must be increased to avoid exceeding damage thresholds, therefore re-absorption of VUV is expected to prove more of a problem. The length of the cell and the location of the lens were chosen so that the $355 \mathrm{~nm}$ beam focused at approximately the centre of the cell, midway between the lens and the entrance of the detector. This minimised the risk of burning tripling cell windows, providing the greatest scope for using higher pump powers.

Theory predicts the power coefficient $G$ is maximum at a value of $b \Delta k=-13$ for tripling a TEM $_{10}$ mode in a single component (see Fig. 3 ). The theoretical value $\left(P_{\mathrm{Xe}, \mathrm{opt}}\right)$ for the optimum Xe pressure in the 

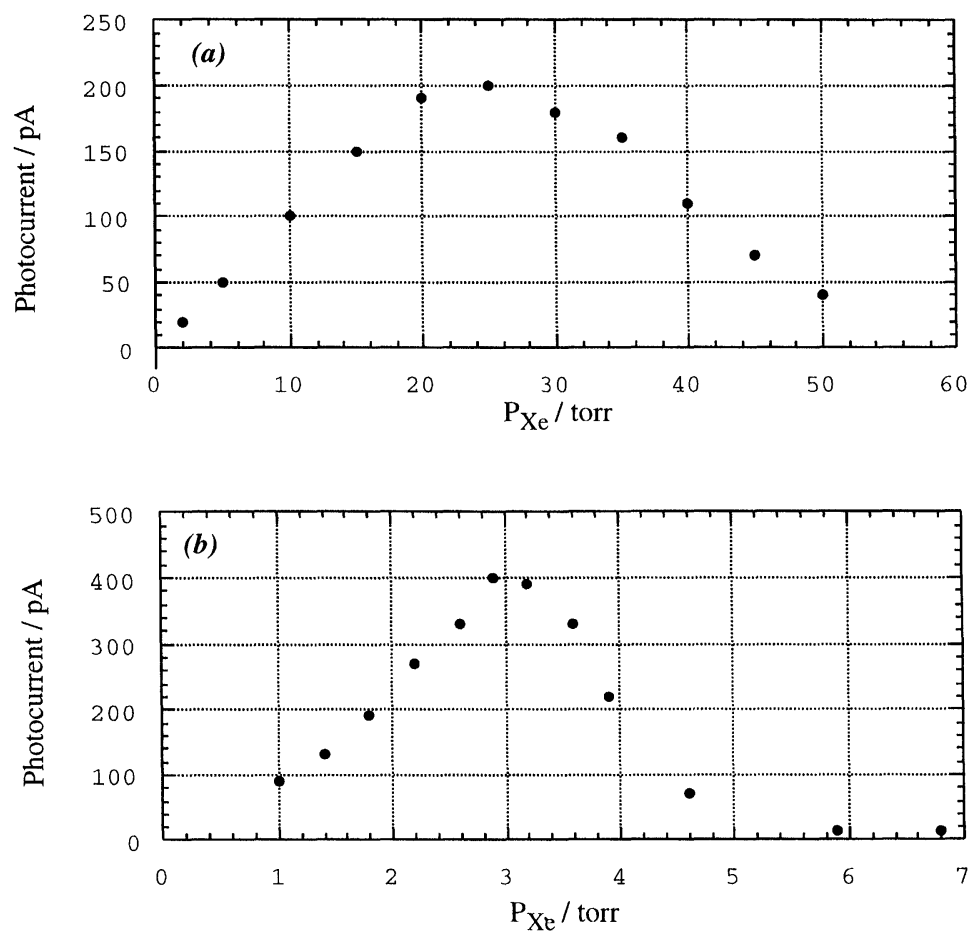

FIGURE 5 THG in xenon with $30 \mathrm{~mJ}$ pulse $\mathrm{e}^{-1}$ at $355 \mathrm{~nm}$ (a) $b=0.2 \mathrm{~cm}, 2 \times 10^{12}$ $\mathrm{W}$ pump power density, (b) $b=2.3 \mathrm{~cm}, 2 \times 10^{11} \mathrm{~W} \mathrm{~cm}^{-2}$ pump power density.

absence of Ar can be calculated from

$$
P_{\mathrm{Xe}, \mathrm{opt}}=b \Delta k_{\mathrm{opt}} \beta / C_{\mathrm{Xe}} b
$$

where,

$$
\begin{aligned}
\beta & =P / N \\
& =3.036 \times 10^{-17} \text { torr } @ 20^{\circ} \mathrm{C} \\
C_{\mathrm{Xe}} & =-6 \times 10^{-17} \mathrm{~cm}^{2}(\text { ref. } 11) \\
b & =\text { confocal parameter }(\mathrm{cm})
\end{aligned}
$$

From eq. (7) $P_{\mathrm{Xe}, \mathrm{opt}}$ is 32.9 torr at $b=0.2 \mathrm{~cm}$ and 2.9 torr at $b=2.3 \mathrm{~cm}$. As the focal length of the lens increases the angle between the three unit vectors $\mathbf{e}_{\mathbf{i}}$ becomes less and the magnitude of the wave vector mismatch required to maintain phase matching decreases. 


\section{Effect of Pump Power on VUV Intensity}

Boyle et al. report the onset of dielectric breakdown at a xenon pressure of 26 torr using $355 \mathrm{~nm}$ TEM $_{10}$ pulses of $>1 \times 10^{10} \mathrm{~W}$ $\mathrm{cm}^{-2}$ [12]. This may explain why the conversion efficiency is observed to peak at about 25 torr xenon in Figure 5(a) rather than at the theoretical value of 32.9 torr using this focal length lens. With the $f_{355}=50 \mathrm{~cm}$ lens the experimental value of $P_{\text {Xe,opt }}$ is in excellent agreement with theory (Fig. 5(b)), suggesting that laser-induced gas breakdown is not a limiting factor using this experimental geometry.

A power study using the $f_{355}=50 \mathrm{~cm}$ lens found the VUV intensity to be linearly dependent on the cube of the UV pulse energy up to the limit of our available pump power density $\left(1 \times 10^{11} \mathrm{~W} \mathrm{~cm}{ }^{-2}\right)$. This suggests that the observed loss in conversion efficiency above 3 torr $\mathrm{Xe}$ using the $b=2.3 \mathrm{~cm}$ lens is not a saturation or breakdown effect.

Laser-induced gas breakdown has an extremely detrimental effect on conversion efficiency since the ionisation of the tripling medium not only reduces the population of ground state atoms, which constitute the nonlinear medium, but the production of photoelectrons introduces an additional large positive dispersion and destroys the phase matching of the mixture. The higher the gas pressure the lower the energy threshold will be for breakdown. Zych et al. report breakdown in 5 torr of $\mathrm{Xe}$ at $1 \times 10^{12} \mathrm{~W} \mathrm{~cm}^{-2}$ at the focus of a Gaussian 355 $\mathrm{nm}$ beam [13]. The mode structure of the DCR-11 laser $\left(\mathrm{TEM}_{10}\right)$ means breakdown thresholds at a given pressure are larger than for $\mathrm{TEM}_{00}$ modes. This is because the intensity profile of the $\mathrm{TEM}_{10}$ beam is more disperse and the peak intensity which occurs at the centre of the Gaussian beam is absent.

\section{Phase Matching}

Figure 6 shows the THG phase matching curve for pure xenon using an $f_{355}=50 \mathrm{~cm}$ lens and $30 \mathrm{~mJ}$ pulse $\mathrm{s}^{-1}$ at $355 \mathrm{~nm}$ and the corresponding curve for 10 torr of Xe mixed with Ar. In the single component medium the conversion efficiency maximises at about 2.7 torr Xe, which is consistent with Figure 5(b). In a two component mixture the optimum Ar:Xe ratio is about 8.5. The peak photocurrent in the two component mixture is a factor 6.2 larger than that in pure 


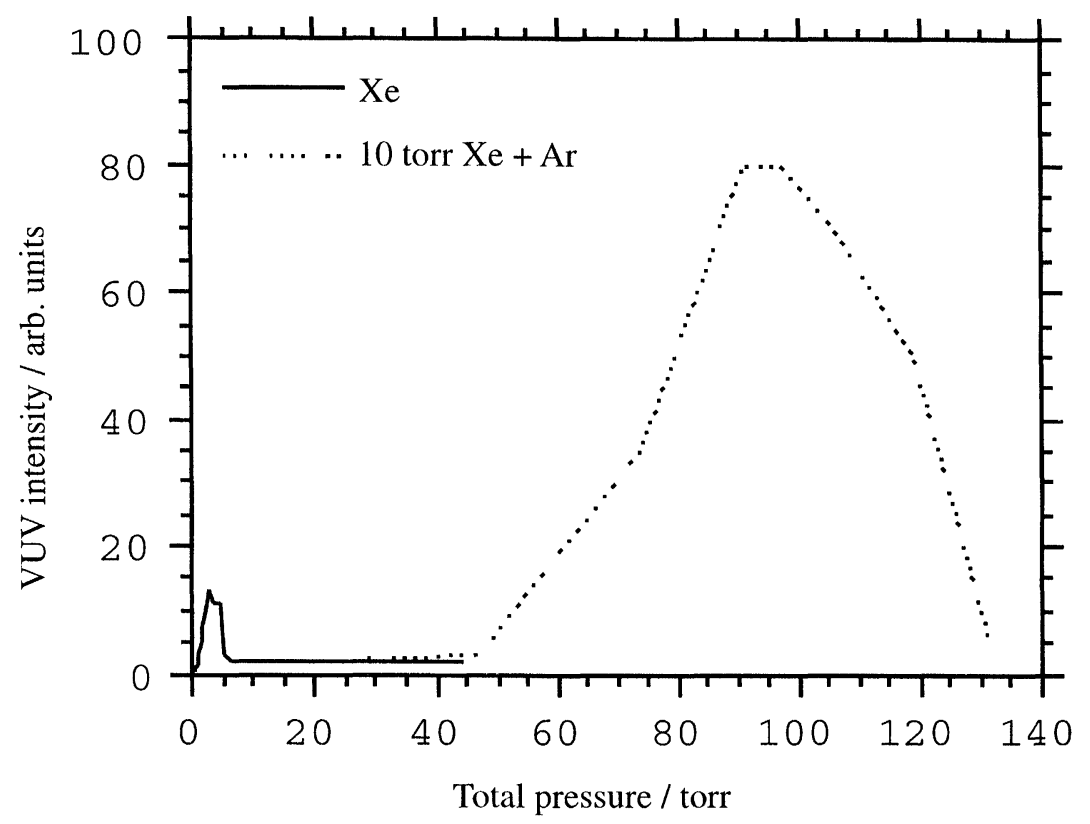

FIGURE 6 Phase matching in xenon and xenon/argon $\left(30 \mathrm{~mJ}\right.$ pulse $\mathrm{s}^{-1}$ at $355 \mathrm{~nm}$, $b=2.3 \mathrm{~cm})$.

xenon, illustrating the increased VUV intensity obtained by increasing the number density of the scattering centres.

The peak photocurrents of $130 \mathrm{pA}$ and $800 \mathrm{pA}$ clearly deviate from the theoretical $N^{2}$ improvement on increasing the Xe pressure by a factor of 3.7. In fact the photocurrent only increases by about half as much as expected. This could be due to the increased re-absorption of VUV at higher pressures. With an $f_{355}=50 \mathrm{~cm}$ lens the breakdown threshold of $1 \times 10^{12} \mathrm{~W} \mathrm{~cm}^{-1}$ in 26 torr $\mathrm{Xe}$ is not reached until pulse energies exceed $200 \mathrm{~mJ}$, so gas breakdown can be ruled out as a loss mechanism in this case.

Figure 7 compares phase matching 5 and 10 torr of Xe with Ar. The optimum VUV intensity is approximately doubled on doubling the $\mathrm{Xe}$ pressure and restoring the optimum phase matching by adding Ar. The optimum values for $P_{\mathrm{Xe}}$ and $P_{\mathrm{Ar}}$ are in excellent agreement with the theory. This supports the assumption that gas breakdown is not occurring in 10 torr Xe. 


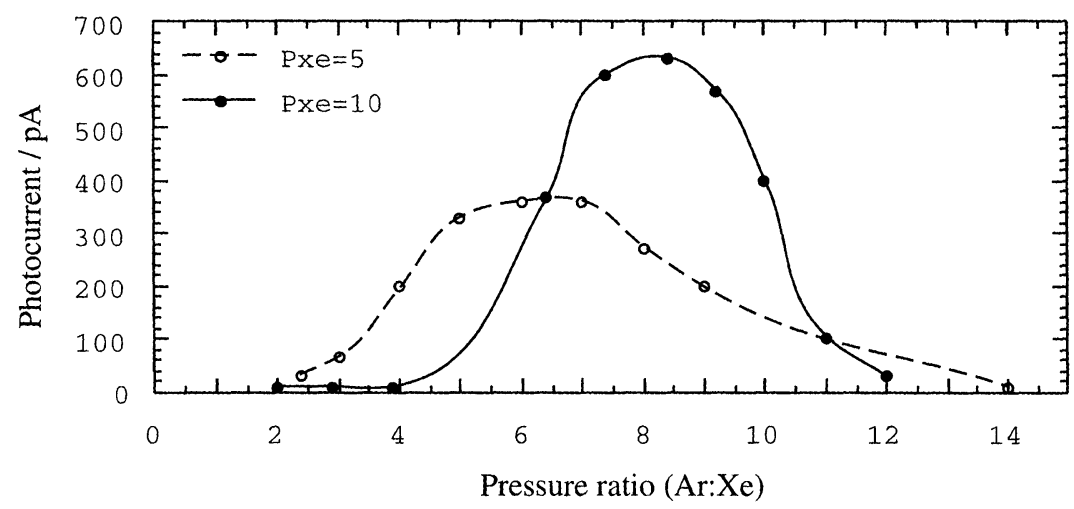

FIGURE 7 Phase matching 5 torr and 10 torr Xe with $\operatorname{Ar}\left(30 \mathrm{~mJ}\right.$ pulse $^{-1}$ at $355 \mathrm{~nm}$, $b=2.3 \mathrm{~cm})$.

The power coefficient $F$ has its largest maximum at $b \Delta k=-11.5$. The corresponding phase matched mixture of $P_{\mathrm{Xe}}$ and $P_{\mathrm{Ar}}$ is given by

$$
P_{\mathrm{Xe}} C_{\mathrm{Xe}}+P_{\mathrm{Ar}} C_{\mathrm{Ar}}=b \Delta k_{\mathrm{opt}} \beta / b
$$

where [11],

$$
C_{\mathrm{Ar}}=5.5 \times 10^{-18} \mathrm{~cm}^{2}
$$

Substituting in a value of $P_{\mathrm{Xe}}=10$ torr gives a value of $P_{\mathrm{Ar}}=81$ torr for the phase matched mixture, which corresponds to a ratio (Ar:Xe) of 8.1:1. The theoretical ratio for a xenon pressure of 5 torr is 5.5 according to eq. (8).

From eq. (8) it can be shown that

$$
P_{\mathrm{Xe}} / P_{\mathrm{Ar}}=\left(\Delta k_{\mathrm{opt}} \beta / C_{\mathrm{Xe}} P_{\mathrm{Ar}}\right)-C_{\mathrm{Ar}} / C_{\mathrm{Xe}}
$$

Clearly the optimum value of $P_{\mathrm{Xe}} / P_{\mathrm{Ar}}$ depends on the pressure. However, at high pressures, $P_{\mathrm{Xe}} / P_{\mathrm{Ar}}$ approaches $-C_{\mathrm{Ar}} / C_{\mathrm{Xe}}$, which corresponds to a ratio (Ar:Xe) of 10.9:1.

As in Figure 6, the improvement in the conversion efficiency is about half that expected from an $N^{2}$ relationship. Theory predicts that the VUV intensity should quadruple on doubling the number density of 
the xenon, provided the phase matching is not disturbed (eq. (3)). The discrepancy between experiment and theory is again attributed to reabsorption of VUV.

It is not known whether the majority of re-absorption is caused by $\mathrm{Xe}$ atoms or some other species. Likely candidates include $\mathrm{Xe}_{2}$ dimers which may be present at significant levels at rare gas pressures in the several hundred torr range. Besides re-absorbing VUV, the production of $\mathrm{Xe}_{2}$ dimers reduces the density of the nonlinear medium (Xe atoms) and provides an additional source of positive dispersion which will affect the overall phase matching. Heating the tripling cell would reduce the dimer population but would also shift the absorption spectrum and so the results of the experiment would be inconclusive. Another possible candidate is residual nitrogen, although this is expected to be present in very low quantities due to the care taken in the construction and operation of the cell.

\section{Summary}

The experiments described above do not constitute an exhaustive optimisation of the conversion process of $355 \mathrm{~nm}$ to $118 \mathrm{~nm}$ light in xenon and argon. They do however identify important parameters in this process and illustrate the means by which a more detailed study may be carried out.

The maximum VUV flux generated to date in our laboratory is $10^{10}$ photons pulse $\mathrm{e}^{-1}$. This was obtained with $1 \times 10^{11} \mathrm{~W} \mathrm{~cm}^{-2}$, at $355 \mathrm{~nm}$, using an $f=50 \mathrm{~cm}$ lens and 10 torr Xe phase matched with Ar. The transmission of $118 \mathrm{~nm}$ through the $\mathrm{MgF}_{2}$ window is estimated at $60 \%$ and the collection efficiency of the detector is assumed to be $10 \%$, on the basis of calibrations performed on similar designs [14].

\section{APPLICATION TO SINGLE PHOTON IONISATION MASS SPECTROMETRY}

\section{Experimental}

For SPI mass spectrometry the tripling cell described above is attached to a Kratos Prism series Time-of-Flight mass spectrometer. The 
ToFMS has been described previously by Scrivener et al. [15]. The $\mathrm{MgF}_{2}$ exit window of the cell provides the vacuum interface to the mass spectrometer. A LiF plano-convex lens mounted in front of the ion source region serves to focus the laser radiation parallel to and 1 $\mathrm{mm}$ above the surface of a sample stub, in the axis of the mass spectrometer.

The focal length of the LiF lens is $17 \mathrm{~cm}$ at $355 \mathrm{~nm}$, and $10 \mathrm{~cm}$ for the $118 \mathrm{~nm}$ beam. The combined "back focal length" can be calculated from eq. (10)

$$
b f l_{\lambda}=f_{2}\left(d-f_{1}\right) /\left(d-\left(f_{1}+f_{2}\right)\right)
$$

where

$$
\begin{aligned}
b f l_{\lambda}= & \text { combined focal length of 2-lens system at wavelength } \lambda \\
& \quad \text { (measured from second lens element) } \\
f_{x}= & \text { focal length of lens }(x) \\
d= & \text { lens separation }
\end{aligned}
$$

Using an $f_{355}=50 \mathrm{~cm}$ lens to focus the UV beam into the tripling cell and a lens separation $d=82 \mathrm{~cm}$, the VUV cross sectional area $\left(A_{118}\right)$ was calculated $1 \times 10^{-3} \mathrm{~cm}^{2}$ in the ion axis of the mass spectrometer. The residual UV beam cross sectional area $\left(A_{355}\right)$ was calculated as $3 \times 10^{-2} \mathrm{~cm}^{2}$, this value was confirmed by obtaining burn profiles on thermal paper. The $118 \mathrm{~nm}$ energy density was estimated to be of the order $0.1 \mu \mathrm{J} \mathrm{cm}^{-2}$ at the ion source region, based on measurements of photocurrents detected in the acetone ionisation chamber.

The sample potential was held at $2.7 \mathrm{kV}$, and the pass energy of the spectrometer optimised for $2.5 \mathrm{keV}$ ions, formed $1 \mathrm{~mm}$ above the sample surface.

\section{Results and Discussion}

\section{Alignment of VUV Beam}

Due to the low efficiency of the frequency conversion process the flux of residual UV photons exiting the tripling cell far exceeds that of 
VUV photons. In order to minimise the MPI background signal and maximise the SPI efficiency it is necessary to carefully align the laser beam in the region of the ion source.

To monitor the alignment proceedure a mixture of hexanes were chosen as analyte molecules. This sample not only displays a different fragmentation pattern for SPI and MPI, but exhibits a large photon absorption cross section at $118 \mathrm{~nm}$ and a high vapour pressure at room temperature so is easily introduced to the system in the gas phase.

Figure 8(a) shows the SPI mass spectrum of a hexanes mixture obtained with the laser beams aligned centrally through the second lens element. In addition to the molecular ion $\left(\mathrm{C}_{6} \mathrm{H}_{14}^{+}\right)$at $\mathrm{m} / \mathrm{z} 86$, fragment ions signals are detected for $\mathrm{C}_{4} \mathrm{H}_{9}^{+}, \mathrm{C}_{3} \mathrm{H}_{7}^{+}$and $\mathrm{C}_{2} \mathrm{H}_{5}^{+}$.
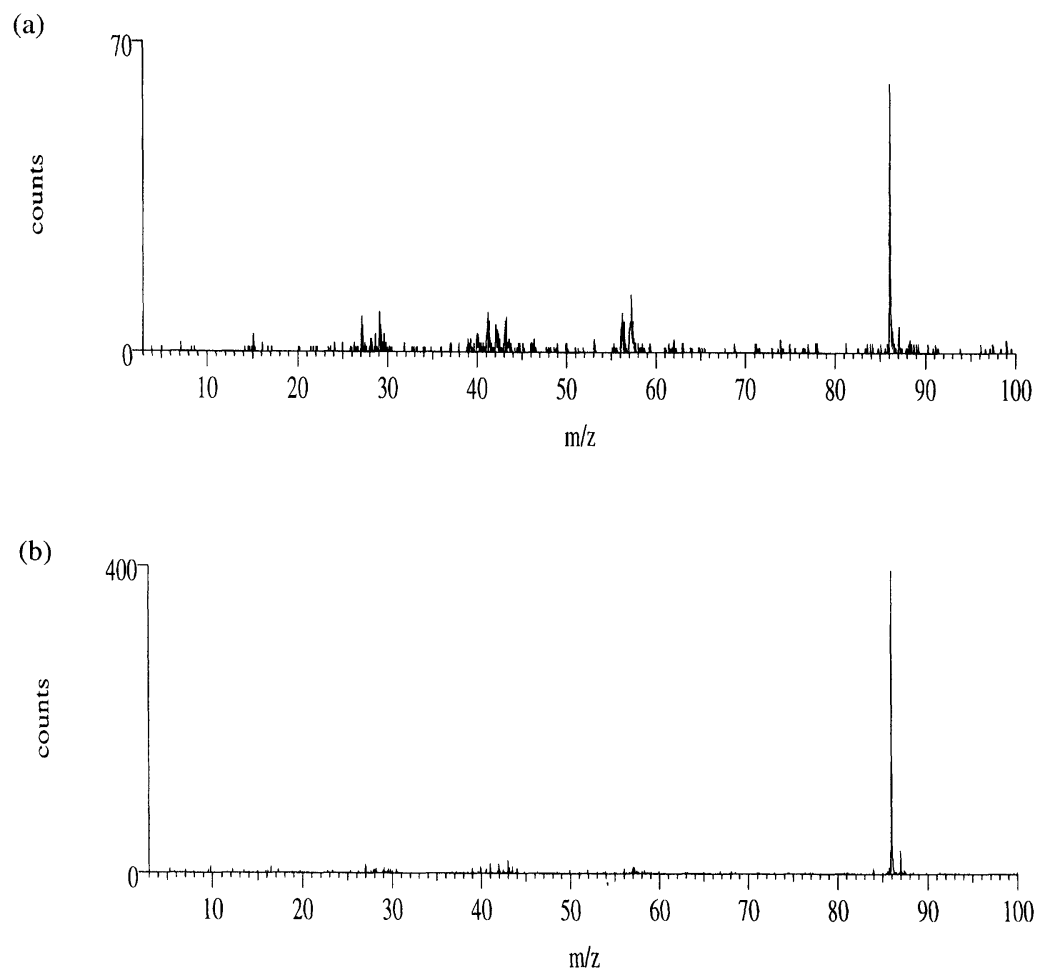

FIGURE 8 SPI mass spectra of hexane $\left(5 \times 10^{-6} \mathrm{mb}\right.$ static pressure) using a) centrally aligned laser beams and $b$ ) non-centrally aligned beams. 
Pumping out the tripling cell results in an almost complete loss of molecular ion signal but has no affect on the intensity of the fragment ions, which therefore constitute an MPI background due to the residual UV $\left(0.6 \mathrm{~J} \mathrm{~cm}^{-2}\right)$.

Figure $8(\mathrm{~b})$ shows the same sample with the photon beam exiting the tripling cell directed through the edge of the LiF lens. The non-central alignment through the lens causes the UV and VUV paths to be spatially dispersed due to the different refractive indices of the lens material at $355 \mathrm{~nm}$ and $118 \mathrm{~nm}$. It can be seen in Figure 8(b) that the parent/fragment ion ratio has increased significantly with respect to Figure 8(a) due to the movement of the residual UV beam out of the ion source region. A similar effect can be achieved by passing the beams at non-normal incident through a plane window.

\section{SPI Mass Spectrometry of Biomolecules}

To evaluate the application of the $118 \mathrm{~nm}$ source to the ionisation of very fragile biomolecules, a series of DL-dipeptides were analysed. These data are briefly introduced here and will be reported and discussed in greater detail elsewhere.

Samples were deposited from methanolic solution onto clean copper stubs and thermally desorbed at approx $150^{\circ} \mathrm{C}$ in the region of the mass spectrometer ion source. A pressure of about $1 \times 10^{-8}$ torr was maintained in the analysis chamber during the experiment. A noncentrally aligned laser beam was employed for maximum MPI supression.

Figure 9 shows the SPI mass spectra of the dipeptide valyl-valine obtained with 3000 laser pulses. The spectrum is clearly dominated by the immonium ion val- $\mathrm{COOH}$ at $\mathrm{m} / \mathrm{z} 72$. This fragmentation is typical of all amino acids under a wide range of photoionisation conditions. The molecular ion at $\mathrm{m} / \mathrm{z} 216$ is clearly seen, albeit $<4 \%$ of the intensity of the base peak. Parent ion intensities at $<10 \%$ of the base peak have been reported in SPI studies on other di- and tri-peptide systems [3]. The structurally significant $\mathrm{M}-\mathrm{NH}_{2} \mathrm{COOH}$ fragment at $\mathrm{m} / \mathrm{z} 155$ is also detected.

The observed fragmentation is extremely unlikely to be the result of the absorption of more than one $118 \mathrm{~nm}(10.5 \mathrm{eV})$ photon due the very low peak flux density of VUV photons $\left(1 \times 10^{19}\right.$ photons $\left.\mathrm{cm}^{-2} \mathrm{~s}^{-1}\right)$. 
The absorption of two VUV photons would deposit $21 \mathrm{eV}$ of energy in the molecule, which is sufficient to ionise carbon and is expected to result in the formation of a high proportion of $C_{1}, C_{2}$ and $C_{3}$ species. The immonium ion is the smallest fragment detected in the val-val SPI mass spectrum. The subsequent absorption of $355 \mathrm{~nm}$ photons from the wings of the residual UV beam cannot be ruled out as a mechanism contributing to fragmentation of ions formed by SPI. The current optical arrangement may not allow complete removal of the UV beam from the ion source region. A suitable filter could be used to reduce the UV flux exiting the tripling cell, but this would inevitably reduce the VUV flux also.

For comparison, Figure 10 shows the MPI mass spectrum of val-val obtained by refocusing the $355 \mathrm{~nm}$ beam to produce an energy density of $670 \mathrm{~J} \mathrm{~cm}^{-2}\left(2 \times 10^{29}\right.$ photons $\mathrm{cm}^{-2} \mathrm{~s}^{-1}$ peak flux density). Val-Val has no aromatic side chain to act as a chromophoric group for photon absorption in the near UV, so multiple photon absorption must proceed via a coherent, nonresonant mechanism. This process requires high photon flux density and often leads to additional photon absorption within the molecular ion manifold and a high degree of subsequent fragmentation. This behaviour can be seen in the val-val MPI mass spectra, which contains ion signals from $C_{1}$ and $C_{2}$ species and in which no fragments larger than the immonium ion where detected under any circumstances.

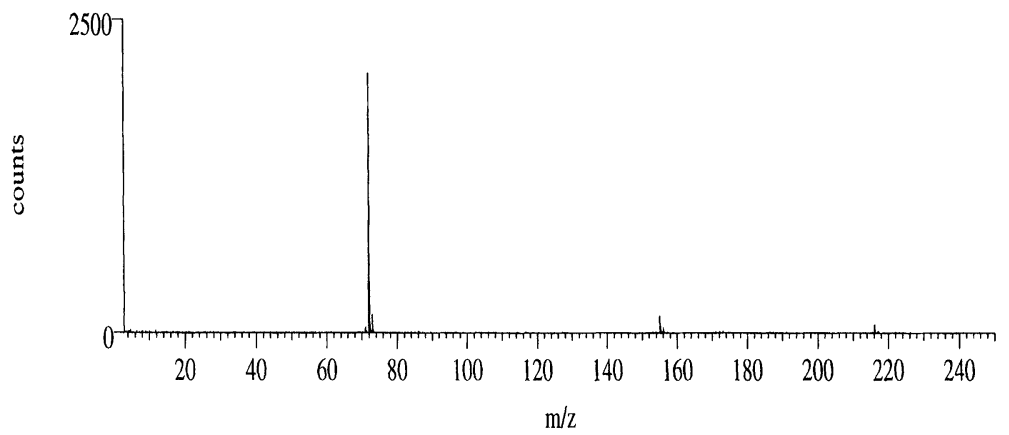

FIGURE 9 SPI mass spectra of Val-Val using $118 \mathrm{~nm}$ photons $\left(0.1 \mu \mathrm{J} \mathrm{cm}{ }^{-2}\right)$. 


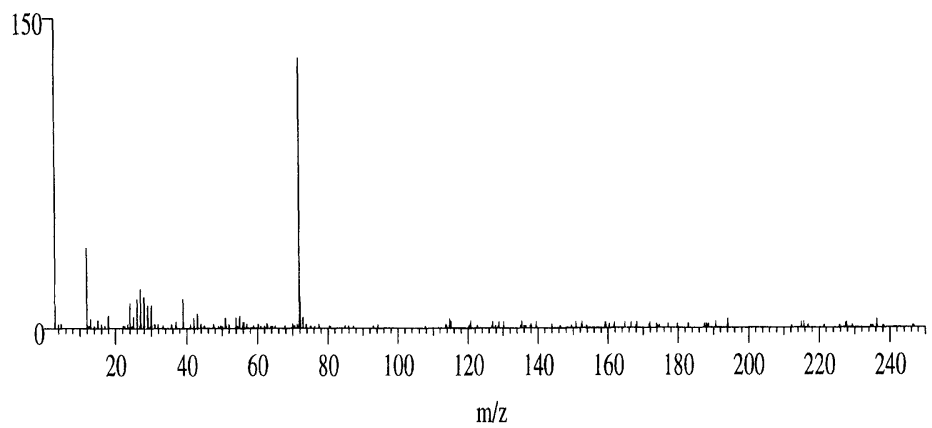

FIGURE 10 MPI mass spectra of Val-Val using $355 \mathrm{~nm}$ photons $\left(670 \mathrm{~J} \mathrm{~cm}^{-2}\right)$.

\section{CONCLUSION}

We have performed a series of experiments to determine the optimum conditions for frequency tripling a $355 \mathrm{~nm}$ laser beam from a Nd:YAG laser in a Xe gas cell. A measure of the absolute number of $118 \mathrm{~nm}$ photons generated is obtained using a home-made acetone ionisation chamber. Power densities up to $1 \times 10^{12} \mathrm{~W} \mathrm{~cm}^{-2}$ at $355 \mathrm{~nm}$ in the region of the nonlinear interaction provide no evidence for laser-induced gas breakdown in $<10$ torr Xe. The optimum Xe pressure for third harmonic generation has been established under two different focussing conditions and agrees well with a theoretical treatment assuming a TEM $_{10}$ pump beam mode structure. An $f=50 \mathrm{~cm}$ focal length lens produces at least twice as much VUV at pump pulse energies of $30 \mathrm{~mJ}$ relative to an $f=15 \mathrm{~cm}$ lens. Experiments with phasematched mixtures of $\mathrm{Xe}$ and Ar indicate that the dependence of the conversion efficiency on the number density of the nonlinear medium is less than predicted by theory, suggesting that re-absorption of VUV is a significant loss mechanism.

Using the laser-generated VUV beam, SPI experiments have been performed in a Time-of-Flight mass spectrometer. The importance of spatially dispersing the VUV and residual UV beams is illustrated using a hexanes sample. The ability of the SPI technique to efficiently produce molecular ions and structurally significant fragments from fragile, non-chromophore containing analytes has been shown using the dipeptide val-val. 


\section{Acknowledgements}

The authors would like to thank the EPSRC for funding this work.

\section{References}

[1] Schühle, U., Pallix, J. B. and Becker, C. H. (1988). J. Vac. Sci. Technol., A 6(3), 936.

[2] Van Bramer, S. E. and Johnston, M. V. (1990). J. Am. Soc. Mass Spectrom., 1, 419.

[3] Köster, C. and Grotemeyer, J. (1992). Organic Mass Spectrom., 27, 463.

[4] Delone, N. B. and Krainov, V. P. (1988). in "Fundamentals of Nonlinear Optics of Atomic Gases", Wiley, New York.

[5] L'Huiller, A., Lompré, L. A., Mainfray, G. and Manus, C. (1991). Adv. At. Mol. Phy., 24, 3315.

[6] Bjorklund, C. (1975). I.E.E.E. J. Quantum Electron., 11, 287.

[7] Steenvoorden, R. J. J. M., Kistemaker, P. G., DeVries, A. E., Michalak, L. and Nibbering, N. M. M. (1991). Int. J. Mass Spectrom. Ion Phys., 107, 475.

[8] Yiu, Y. M., McIlrath, T. J. and Mahon, R. (1979). Phys. Rev., A. 20(6), 2470.

[9] Van Bramer, S. E. and Johnston, M. V. (1992). Appl. Spec., 46(2), 255.

[10] Cotter, D. (1979). Opt. Commun., 31, 379.

[11] Mahon, R., McIlrath, T. J., Myerscough, V. P. and Koopman, D. W. (1979). I.E.E.E.J. Quantum Electron., 15, 444.

[12] Boyle, J. A., Pfefferle, L., Lobue, J. and Colson, S. (1990). Comb. Sci. Technol., 70, 187.

[13] Zych, L. J. and Young, J. F. (1978). I.E.E.E.J. Quantum Electron., 14, 147.

[14] Stober, A. K., Scolnik, R. and Hennes, J. P. (1963). Appl. Optics., 2(7), 735.

[15] Scrivener, E., Wilson, R. C. and Vickerman, J. C. (1995). Surf. Interface Anal., 23, 623. 